Published in final edited form as:

Curr Opin Biotechnol. 2014 February ; 0: 24-29. doi:10.1016/j.copbio.2013.08.007.

\title{
Animal microsurgery using microfluidics
}

\author{
Jeffrey N. Stirman ${ }^{1,2,3}$, Bethany Harker ${ }^{4}$, Hang Lu ${ }^{5}$, and Matthew M. Crane ${ }^{6,{ }^{*}}$ \\ ${ }^{1}$ Carolina Institute for Developmental Disabilities, University of North Carolina, Chapel Hill, North \\ Carolina, USA \\ ${ }^{2}$ Neuroscience Center, University of North Carolina, Chapel Hill, North Carolina, USA \\ ${ }^{3}$ Department of Cell and Molecular Physiology, University of North Carolina, Chapel Hill, North \\ Carolina, USA \\ ${ }^{4}$ Wellcome Trust Centre for Cell Biology, University of Edinburgh, Edinburgh, UK \\ ${ }^{5}$ School of Chemical \& Biomolecular Engineering, Georgia Institute of Technology, Atlanta, GA, \\ USA \\ ${ }^{6}$ SynthSys, University of Edinburgh, Edinburgh, UK
}

\begin{abstract}
Small multicellular genetic organisms form a central part of modern biological research. Using these small organisms provides significant advantages in genetic tractability, manipulation, lifespan and cost. Although the small size is generally advantageous, it can make procedures such as surgeries both time consuming and labor intensive. Over the past few years there have been dramatic improvements in microfluidic technologies that enable significant improvements in microsurgery and interrogation of small multicellular model organisms.
\end{abstract}

\section{Introduction}

Small multicellular model organisms such as D. rerio, D. melanogaster, or C. elegans are central to modern biological research; not only do they have significant advantages in genetic tractability and lifespan, but investigations can be performed with a large number of animals or conditions for the same cost and time that merely preliminary studies in mammalian models would afford. Because of the organism size and genetic tractability, there is a wealth of information from the large-scale genome-wide studies that have been performed with each of these models.

Recent advances in the field of microfluidics have made the manipulation of fluid volumes on the same length and volume scales as the organisms themselves relatively routine. Although handling multicellular organisms is a complex task, significant microfluidic advances promise to allow fully automated handling and completion of challenging surgical manipulations such as laser axotomy and microinjection. At the microfluidic scale fluid flow is laminar and predictable, allowing consistent sample handling. Also, due to the large surface area to volume ratio, dissolved gases and chemicals can be allowed to diffuse in a

\footnotetext{
(C) 2013 Elsevier Ltd. All rights reserved.

*corresponding author: mcrane2@staffmail.ed.ac.uk.
}

Publisher's Disclaimer: This is a PDF file of an unedited manuscript that has been accepted for publication. As a service to our customers we are providing this early version of the manuscript. The manuscript will undergo copyediting, typesetting, and review of the resulting proof before it is published in its final citable form. Please note that during the production process errors may be discovered which could affect the content, and all legal disclaimers that apply to the journal pertain. 
rapid manner allowing rapid changes of reagents. The small thermal mass of these fluids also makes it possible to precisely cool small fluid volumes rapidly. Most microfluidic technologies are still being developed in primarily engineering labs, through the use of rapid prototyping and soft lithography[1,2]. As the technology is gradually being improved, however, it is adopted by increasingly more biology labs to pursue projects that would have previously been either impractically difficult, or impossible.

Much of the pioneering work on microfluidic systems for small animals has focused on consistent sample handling, and providing robust platforms for imaging. This is an important area, but because of the breadth of other reviews covering this topic[3-6], those works will not be discussed here unless they directly relate to the topic of surgery and micro-manipulation (Figure 1). Platforms for robust and automated imaging will, however, act as significant enabling technologies for the development of high-throughput microsurgery systems.

\section{Laser Ablation and Axotomy}

One major advantage small multicellular models offer is their simplified neuronal systems. A primary goal of studying these animals is to develop comprehensive models of how genes influence neurons and how these neurons influence behavior. To this end, optical surgery has become a common tool wherein individual neurons of an animal are ablated[7] (Figure 1A). This is used to remove specific nodes on a neural network and observe how the organism responds. Laser ablation of cells is also commonly used to dissection tissue samples. More recently, the creation of femtosecond lasers and their extremely fast pulse rate has allowed subcellular features within cells, such as axons, cytoskeletal filaments, or stress fibers, to be ablated without harming surrounding tissues[8-10] (Figure 1B). Rather than just removing entire nodes of a network, it is now possible to destroy part of an axon, removing individual connections, and has been used to study the genetics underlying axonal regeneration[11-16].

Performing cellular, or subcellular optical surgery requires a perfectly immobile animal such that the points of interest (along the axon) can be identified and then optically targeted for ablation. This is typically done by anaesthetizing the animal on an agar pad, and using a fluorescent reporter to label the axon of interest, which is manually targeted[7]. In addition to being a labor intensive process, variations in the time animals are anesthetized could have potential effects on the behavioral phenotype or regeneration time[17]. To address these weaknesses, several microfluidic systems have been developed to improve the handling of these organisms, and allow precise targeting of subcellular features[13-15,17-21].

These systems can be separated into those that are largely automated, and those that improve the sample handling speed and reliability but rely on manual processing to a large degree. Generally speaking, the systems focused on C. elegans are more developed compared with those of zebrafish and Drosophila. Because of the early work on imaging automated imaging systems for C. elegans[22,23], laser microsurgery for worms proceeded at a faster pace. Highly automated systems for the axotomy of zebrafish embryos, however, have recently been demonstrated[18]. It should be noted that all of the automated systems have custom designed components, and thus are much more difficult to operate outside of the setting in which they were developed. As a result, to our knowledge, the automated laser microsurgery systems have only been used by the labs where they were developed.

The majority of microfluidic surgery systems for C. elegans have focused on improving the speed and ease of laser axotomy. This is both due to the high interest in axotomy systems recently, and the fact that axotomy is often performed on L4 or adult animals, whereas laser ablation is typically performed on L1 or L2 stage animals. The small size of these animals 
make it much more difficult to develop microfluidic devices for. To date, only a single microsystem has been developed and used for laser ablation of neurons[21].

The microfluidic systems for laser surgery of $C$. elegans use one of either two immobilization mechanisms: transient cooling, or physical constriction. Using the high surface area to volume ratio of microfluidic systems, Chung et al.[21] developed a system for laser ablation of cells where animals are transiently cooled to $\sim{ }^{\circ} \mathrm{C}$ (Figure $2 \mathrm{~A}$ ). Animals are only cooled and immobilized for the few seconds it takes to ablate the cells of interest, and then moved back to ambient temperatures. Physical constriction systems are either 1) passive systems wherein animals are forced into very small channels that prevent movement[14,24] (Figure 2D), or 2) active systems that employ a microfluidic valve to reversibly constrict and immobilize animals[15,17,20,25,26] (Figure 2B,E). Passive trapping and immobilization is less flexible in that only a fixed number of animals on a chip can be processed, and thus cannot be integrated into to extremely high-throughput automated systems. It also requires fewer active components and is thus less prone to failure and can be more easily adapted into non-engineering research labs. Active constriction is sometimes used in conjunction with $\mathrm{CO}_{2}$ gas diffusion to reduce animal movement[26] (Figure 2E). Neither cooling nor physical constriction appears to result in any observable abnormal developmental or behavioral phenotypes[17,21,22].

Laser microsurgery has been primarily limited to C. elegans, but is increasingly being employed in zebrafish[18,19,27] and fruit fly embryos[28]. Recent systems to allow largescale trapping and ordering of embryos of both organisms have made it possible to perform optical microsurgery on chip (Figure 2F). Similar to C. elegans systems, these can be divided into passive devices used to trap and orient animals, and active devices used for high-throughput automated processes. The Yanik lab has developed a system for performing automated axotomy on zebrafish larvae, which has been used to process several hundred animals at a rate of $<20$ s per animal[ 18,19$]$. In contrast to most microfluidic devices that employ PDMS and soft-lithography, this system uses glass capillary tubes. The first microfluidic device that allows trapping of living drosophila larvae, and monitoring them after neuronal injury was recently developed[28]. This device traps larvae while providing a stable nutrient environment, and immobilizes them as intact, un-anaesthetized animals. Larvae are immobilized by a combination of physical constriction and $\mathrm{CO} 2$ gas diffusion through a microfluidic valve[28], similar to work the Chronis lab previously published in $C$. elegans[26]. Animals were demonstrated to recover within 30 seconds following immobilization, and the chips were used to study the rate of axonal transport in recently damaged neuronal systems. Larvae had an $85 \%$ survival rate after immobilization processes over 10 hours of repeated immobilization. Recent work on developing large-scale arrays of fixed Drosophila embryos for imaging $[29,30]$ and zebrafish embryos [31,32] might be extended in a similar fashion to allow easily translatable systems for large-scale regeneration studies (Fig 2C).

\section{Optical Interrogation}

The recent development of optogenetic techniques has revolutionized neuroscience research. These optical techniques allow interrogation of neuronal circuits in ways that would have previously required invasive surgery and patch clamping (Figure 1D). Cells expressing specific rhodopsins can be de- or hyper-polarized depending the type of rhodopsins expressed and the wavelength of light that they are exposed to. This is a powerful, noninvasive experimental method that is rapidly replacing many surgical alternatives. Few systems are fully developed that integrate both microfluidic environments and optogenetic manipulation, but the separate development of highly controlled environments for behavioral responses, and optical control systems makes the trend in research clear. 
A microfluidic system has been developed that allows high-throughput screening of genes affecting synaptic function in C. elegans[33]. Animals containing ChR2 were processed in microfluidic device, and motor neurons activated by exposure to blue light. By measuring the degree of contraction, the researchers were able to identify genes whose absence reduced the activation strength of neuronal depolarization. Another microfluidic system was recently used in conjunction with optogenetics to study proprioception and wave propagation along the $C$. elegans body[34]. By trapping animals in precise configurations, and then activating or inactivating specific body wall muscles, the researchers were able to identify how coordinated wave patters are generated to allow forward motion. Using a microfluidic valve that would push the animal from the side, the curvature of the animal could be dynamically altered and the effect on the motor system observed. Recent work on optical systems has demonstrated single cell multispectral optical targeting of moving C. elegans on agar plates[35-37]. When combined with noninvasive monitoring of neuronal responses, these technologies offer the ability to effectively perform dynamic, and reversible laser ablation that can provide highly insightful information about neural networks[38,39]. These optical systems are likely to be combined with microfluidic devices capable of providing a precisely controlled chemical environment[40].

\section{Mechanical Manipulations}

One of the most labor-intensive aspects of model organism research is the creation of transgenic strains, or knocking down individual genes by microinjection (Figure 1C). With the capacity to perform high-resolution, automated imaging of multicellular model organisms, the primary bottleneck for many large-scale projects is the ability to transform, introduce RNAi, or morpholino to animals quickly and with high success rates. This need is further compounded by the need to have transgenic strains for optogenetic experiments. Targeting specific subsets of neurons with different rhodopsins requires tedious and laborious transformations that are significant hindrances to current research practices.

Automated microinjections systems have long been of interest [41], but have failed to receive the same level of attention as imaging and axotomy systems. Part of the challenge for microinjection systems is that the materials commonly used for microfluidic devices are too soft to puncture cell walls or epidermal layers. This means that development is done with hybrid systems composed of multiple materials, which can be more time consuming and expensive. A fully automated system for zebrafish embryos has been developed that uses macro-scale traps and conventional micropipette injection methods[42]. Recently, a combined PDMS, microneedle microfluidic system was developed that uses electroosmotic flow for reagent introduction[43]. By employing EOF, they were able to achieve improved dosage control and improved transfection rates. An automated system for drosophila embryos was just recently presented that employs a pyrex-silicon-pyrex device for injection[44]. In contrast with the other systems that use pulled glass capillary needles, this uses a micromachined silicon nitride injector. This system was used to automatically inject hundreds of embryos for both transformation and with RNAi. This system could be used to perform significantly larger studies of gene expression and roles than previously possible.

\section{Future Directions and Outlook}

One of the significant hurdles facing researchers trying to perform laser microsurgery is the challenge of identifying and ablating the targets of interest (with minimal damage to the surrounding tissue). The majority of automation has been done thus far using relatively simple segmentation methods that work to threshold and identify targets using straightforward heuristics that can make them prone to errors and require recoding for each new target of interest $[18,19,21]$. Computer vision methods, however, have been integrated into small animal microfluidic systems for identification of complex phenotypes with 
increasingly regularity and complexity[45]. Higher-level computer vision approaches offer the potential to identify targets of interest with ease. Over the past few years significant work has been done to create a comprehensive computer atlas of cell positions and lineage[46-49]. Combining these digital atlases with the ablation and axotomy systems could researchers to merely enter cell names into a program, and then ablate or cut at will.

The ability to acquire large numbers of animals with different combinations of ablated cells is likely to increase the reliance on computer-aided phenotyping. Combining computer vision with statistical analysis has the potential to detect extremely subtle phenotypes with high confidence[45,50]. High-content phenotyping will be critical to understanding the subtle information transfer between neurons. In the future, optical control is likely to focus on the development of microfluidic systems for precise environmental and chemical control[40,51], optical system to silence or activate specific neurons in real-time[35,36], and computer vision to analyze, dissect, and understand the complex behavioral phenotypes[50]. The technological hurdles facing this development are significant, but when accomplished will profoundly expand the opportunities and understanding of neuroscience.

Ordering and manipulation of large embryos will allow higher throughput optical manipulations of larger and more complex model organisms. Microinjection is still a ratelimiting step for large systemic studies of model organisms. This is a research area where microfluidic systems have demonstrated significant potential, but have failed to fully meet the needs. Hopefully in the future technologies will be developed that will be robust enough to allow the development of commercial systems that will make it possible to produce hundreds of transgenic strains within a relatively short amount of time, and for an order of magnitude less than current costs.

The long-term trend is the increasing integration of computer science, microfluidics and biology. Due to the market size, it is improbable many of the systems discussed will be commercialized and thus they are unlikely to have robust support systems. This means labs will have form collaborations to develop and properly use these tools, and will continue the cross pollination between engineering and biological research. Most of the experimental systems discussed in this review paper have been developed in isolation, but are gradually being applied to answer biological questions that would have been intractable without the new methodologies.

\section{References}

1. Xia Y, Whitesides G. Soft lithography. Ann Rev Mater Sci. 1998; 28:153-184.

2. Whitesides GM. The origins and the future of microfluidics. Nature. 2006; 442:368-373. [PubMed: 16871203]

3. Crane MM, Chung K, Stirman J, Lu H. Microfluidics-enabled phenotyping, imaging, and screening of multicellular organisms. Lab on a chip. 2010; 10:1509-1517. [PubMed: 20383347]

4. Ben-Yakar A, Chronis N, Lu H. Microfluidics for the analysis of behavior, nerve regeneration, and neural cell biology in C. elegans. Current opinion in neurobiology. 2009; 19:561-567. [PubMed: 19896831]

5. Yanik MF, Rohde CB, Pardo-Martin C. Technologies for micromanipulating, imaging, and phenotyping small invertebrates and vertebrates. Annual review of biomedical engineering. 2011; 13:185-217.

6. Hwang H, Lu H. Microfluidic tools for developmental studies of small model organismsnematodes, fruit flies, and zebrafish. Biotechnology journal. 2012

7. Fang-Yen C, Gabel CV, Samuel ADT, Bargmann CI, Avery L. Laser microsurgery in Caenorhabditis elegans. Methods in cell biology. 2012; 107:177-206. [PubMed: 22226524] 
8. Yanik MF, Cinar H, Cinar HN, Chisholm AD, Jin Y, Ben-Yakar A. Neurosurgery: functional regeneration after laser axotomy. Nature. 2004; 432:822-822. [PubMed: 15602545]

9. Heisterkamp A, Maxwell IZ, Mazur E, Underwood JM, Nickerson JA, Kumar S, Ingber DE. Pulse energy dependence of subcellular dissection by femtosecond laser pulses. Opt Express. 2005; 13:3690-3696. [PubMed: 16035172]

10. Shen N, Datta D, Schaffer CB, LeDuc P, Ingber DE, Mazur E. Ablation of cytoskeletal filaments and mitochondria in cells using a femtosecond laser nanoscissor. Mechanics and Chemistry of Biosystems. 2005; 2:17-26. [PubMed: 16708469]

11. Chen L, Wang Z, Ghosh-Roy A, Hubert T, Yan D, O'Rourke S, Bowerman B, Wu Z, Jin Y, Chisholm AD. Axon regeneration pathways identified by systematic genetic screening in $\mathrm{C}$. elegans. Neuron. 2011; 71:1043-1057. [PubMed: 21943602]

12. Byrne AB, Edwards TJ, Hammarlund M. In vivo laser axotomy in C. elegans. Journal of visualized experiments: JoVE. 2011:1-6.

13. Neumann B, Nguyen KCQ, Hall DH, Ben-Yakar A, Hilliard Ma. Axonal regeneration proceeds through specific axonal fusion in transected C. elegans neurons. Developmental dynamics. 2011; 240:1365-1372. [PubMed: 21416556]

14. Pinan-Lucarre B, Gabel CV, Reina CP, Hulme SE, Shevkoplyas SS, Slone RD, Xue J, Qiao Y, Weisberg S, Roodhouse K, et al. The Core Apoptotic Executioner Proteins CED-3 and CED-4 Promote Initiation of Neuronal Regeneration in Caenorhabditis elegans. PLoS Biol. 2012; 10:e1001331. [PubMed: 22629231]

15. Samara C, Rohde CB, Gilleland CL, Norton S, Haggarty SJ, Yanik MF. Large-scale in vivo femtosecond laser neurosurgery screen reveals small-molecule enhancer of regeneration. Proceedings of the National Academy of Sciences of the United States of America. 2010; 107:18342-18347. [PubMed: 20937901]

16. Nix P, Hisamoto N. Axon regeneration requires coordinate activation of $\mathrm{p} 38$ and JNK MAPK pathways. Proceedings of the National Academy of Sciences. 2011

17. Guo S, Bourgeois F, Chokshi T, Durr N, Hilliar M, Chronis N, Ben-Yakar A. Femtosecond laser nanoaxotomy lab-on-a-chip for in vivo nerve regeneration studies. Nature Methods. 2008; 5:531533. [PubMed: 18408725]

18**. Chang T-Y, Pardo-Martin C, Allalou A, Wählby C, Yanik MF. Fully automated cellularresolution vertebrate screening platform with parallel animal processing. Lab on a chip. 2012; 12:711-716. Improving on a previous study, the authors demonstrate a fully automated system for zebrafish axotomy. This system automatically moves zebrafish larvae from 96-well plates into a microfluidic system where automated microsurgery is performed. [PubMed: 22159032]

19. Pardo-Martin C, Chang T-Y, Koo BK, Gilleland CL, Wasserman SC, Yanik MF. High-throughput in vivo vertebrate screening. Nature methods. 2010; 7:634-636. [PubMed: 20639868]

20. Zeng F, Rohde CB, Yanik MF. Sub-cellular precision on-chip small-animal immobilization, multiphoton imaging and femtosecond-laser manipulation. Lab on a chip. 2008; 8:653-656. [PubMed: 18432331]

21. Chung K, Lu H. Automated high-throughput cell microsurgery on-chip. Lab on a chip. 2009; 9:2764-2766. [PubMed: 19967110]

22. Chung K, Crane MM, Lu H. Automated on-chip rapid microscopy, phenotyping and sorting of C. elegans. Nature Methods. 2008; 5:637-643. [PubMed: 18568029]

23. Crane MM, Chung K, Lu H. Computer-enhanced high-throughput genetic screens of C. elegans in a microfluidic system. Lab on a chip. 2009; 9:38-40. [PubMed: 19209332]

24. Hulme SE, Shevkoplyas SS, Apfeld J, Fontana W, Whitesides GM. A microfabricated array of clamps for immobilizing and imaging C. elegans. Lab on a chip. 2007; 7:1515-1523. [PubMed: 17960280]

25. Steinmeyer JD, Gilleland CL, Pardo-Martin C, Angel M, Rohde CB, Scott Ma, Yanik MF. Construction of a femtosecond laser microsurgery system. Nature protocols. 2010; 5:395-407.

26. Chokshi TV, Ben-Yakar A, Chronis N. CO2 and compressive immobilization of C. elegans onchip. Lab on a chip. 2009; 9:151-157. [PubMed: 19209348] 
27. Kohli V, Elezzabi AY. Laser surgery of zebrafish (Danio rerio) embryos using femtosecond laser pulses: optimal parameters for exogenous material delivery, and the laser's effect on short-and long-term development. BMC biotechnology. 2008; 8:7. [PubMed: 18230185]

28**. Ghannad-Rezaie M, Wang X, Mishra B, Collins C, Chronis N. Microfluidic chips for in vivo imaging of cellular responses to neural injury in Drosophila larvae. PloS one. 2012; 7:e29869. The authors demonstrate a system to reversibly immobilize drosophila larvae for laser axotomy and monitoring. Following the surgery, the animal can be repeatedly immobilized for high resolution imaging and monitoring of the recovery process. [PubMed: 22291895]

29. Levario TJ, Zhan M, Lim B, Shvartsman SY, Lu H. Microfluidic trap array for massively parallel imaging of Drosophila embryos. Nature protocols. 2013; 8:721-736.

30. Chung K, Kim Y, Kanodia J, Gong E. A microfluidic array for large-scale ordering and orientation of embryos. Nature methods. 2010:8.

31*. Bischel LL, Mader BR, Green JM, Huttenlocher A, Beebe DJ. Zebrafish Entrapment By Restriction Array (ZEBRA) device: a low-cost, agarose-free zebrafish mounting technique for automated imaging. Lab on a chip. 2013:1732-1736. The authors demonstrate a simple microfluidic system for trapping and aligning zebrafish embryos. This is an extremely promising method to ease adoption of microfluidic devices for laser microsurgery by non-engineering labs. [PubMed: 23503983]

32*. Choudhury D, van Noort D, Iliescu C, Zheng B, Poon K-L, Korzh S, Korzh V, Yu H. Fish and Chips: a microfluidic perfusion platform for monitoring zebrafish development. Lab on a chip. 2012; 12:892-900. The authors grow zebrafish embryos in a microfluidic perfusion system, while exposed to multiple drug concentrations. The perfusion system exposes fragile embryos to reduced shear stresses, and allows them to be grown and imaged over multiple days. [PubMed: 22146879]

33. Stirman JN, Brauner M, Gottschalk A, Lu H. High-throughput study of synaptic transmission at the neuromuscular junction enabled by optogenetics and microfluidics. Journal of neuroscience methods. 2010; 191:90-93. [PubMed: 20538016]

34**. Wen Q, Po MD, Hulme E, Chen S, Liu X, Kwok SW, Gershow M, Leifer AM, Butler V, FangYen C, et al. Proprioceptive coupling within motor neurons drives C. elegans forward locomotion. Neuron. 2012; 76:750-761. The authors use microfluidic devices to create a controlled environment where animals can be optically interrogated to decipher neuronal responses in ways that would be impossible with freely moving animals. [PubMed: 23177960]

35. Leifer AM, Fang-Yen C, Gershow M, Alkema MJ, Samuel ADT. Optogenetic manipulation of neural activity in freely moving Caenorhabditis elegans. Nature methods. 2011; 8:147-152. [PubMed: 21240279]

36. Stirman JN, Crane MM, Husson SJ, Gottschalk A, Lu H. A multispectral optical illumination system with precise spatiotemporal control for the manipulation of optogenetic reagents. Nature protocols. 2012; 7:207-220.

37. Stirman JN, Crane MM, Husson SJ, Wabnig S, Schultheis C, Gottschalk A, Lu H. Real-time multimodal optical control of neurons and muscles in freely behaving Caenorhabditis elegans. Nature methods. 2011; 8:153-158. [PubMed: 21240278]

38. Guo ZV, Hart AC, Ramanathan S. Optical interrogation of neural circuits in Caenorhabditis elegans. Nature methods. 2009; 6:891-896. [PubMed: 19898486]

39. Kocabas A, Shen C-H, Guo ZV, Ramanathan S. Controlling interneuron activity in Caenorhabditis elegans to evoke chemotactic behaviour. Nature. 2012; 490:273-277. [PubMed: 23000898]

40. Albrecht DR, Bargmann CI. High-content behavioral analysis of Caenorhabditis elegans in precise spatiotemporal chemical environments. Nature methods. 2011; 8:599-605. [PubMed: 21666667]

41. Lee S, Jeong W, Beebe DJ. Microfluidic valve with cored glass microneedle for microinjection. Lab on a chip. 2003; 3:164-167. [PubMed: 15100768]

42. Wang W, Liu X, Gelinas D, Ciruna B, Sun Y. A fully automated robotic system for microinjection of zebrafish embryos. PloS one. 2007; 2:e862. [PubMed: 17848993]

43. Noori A, Selvaganapathy PR, Wilson J. Microinjection in a microfluidic format using flexible and compliant channels and electroosmotic dosage control. Lab on a chip. 2009; 9:3202-3211.

[PubMed: 19865726] 
44**. Delubac D, Highley CB, Witzberger-Krajcovic M, Ayoob JC, Furbee EC, Minden JS, Zappe S. Microfluidic system with integrated microinjector for automated Drosophila embryo injection. Lab on a chip. 2012; 12:4911-4919. The authors demonstrate a system for automated microinjection in drosophila embryos. The system automatically separates embryo clusters, aligns individual embryos to a silicon nitride microneedle, and injects them with the reagent of interest. [PubMed: 23042419]

45*. Crane M, Stirman J, Ou C, Kurshan P. Autonomous screening of C. elegans identifies genes implicated in synaptogenesis. Nature Methods. 2012:9. The authors screened thousands of animals autonomously and detected novel mutants based on subcellular features. This is a good demonstration of the powers of computer vision and machine learning when combined with microfluidics.

46. Long F, Peng H, Liu X, Kim SK, Myers E. A 3D digital atlas of C. elegans and its application to single-cell analyses. Nature methods. 2009; 6:667-672. [PubMed: 19684595]

47. Murray J, Bao Z, Boyle T, Boeck M. Automated analysis of embryonic gene expression with cellular resolution in C. elegans. Nature .... 2008; 5:703-709.

48. Peng H, Ruan Z, Long F, Simpson JH, Myers EW. V3D enables real-time 3D visualization and quantitative analysis of large-scale biological image data sets. Nat Biotech. 2010; 28:348-353.

49. Ronneberger O, Liu K, Rath M, Rue D, Mueller T, Skibbe H, Drayer B, Schmidt T, Filippi A, Nitschke R, et al. ViBE-Z: a framework for 3D virtual colocalization analysis in zebrafish larval brains. Nat Meth. 2012; 9:735-742.

50. Brown AEX, Yemini EI, Grundy LJ, Jucikas T, Schafer WR. A dictionary of behavioral motifs reveals clusters of genes affecting Caenorhabditis elegans locomotion. Proceedings of the National Academy of Sciences of the United States of America. 2013; 110:791-796. [PubMed: 23267063]

51. Gershow M, Berck M, Mathew D, Luo L, Kane Ea, Carlson JR, Samuel ADT. Controlling airborne cues to study small animal navigation. Nature methods. 2012; 9:290-296. [PubMed: 22245808] 


\section{Highlights}

- The state of microfluidic integration with microsurgery for multicellular models

- Microfluidic methods offer significantly higher throughput and control

- Techniques are increasingly robust and transferable to non-engineering labs

- Integration is allowing large-scale experiments that were previously impossible 
A)

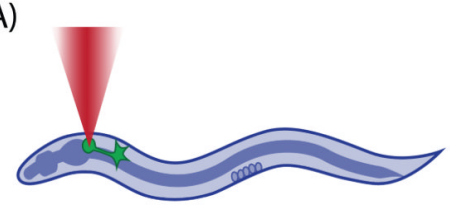

C)

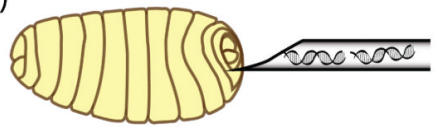

B)

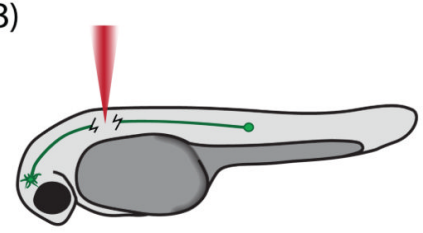

D)

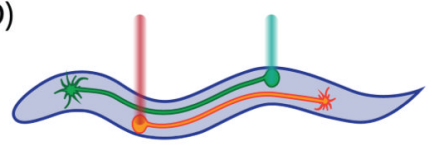

Figure 1.

Microsurgery and micromanipulations methods for animals employing microfluidics. A) Laser ablation of whole cells for behavioral phenotyping and neuroscience. B) Axotomy for studies of axonal regeneration and the drugs and genes involved. C) Microinjection of embryos and larvae for transfection and RNAi knockdowns. D) Optical interrogation of neural circuits using optogenetics. 
A)

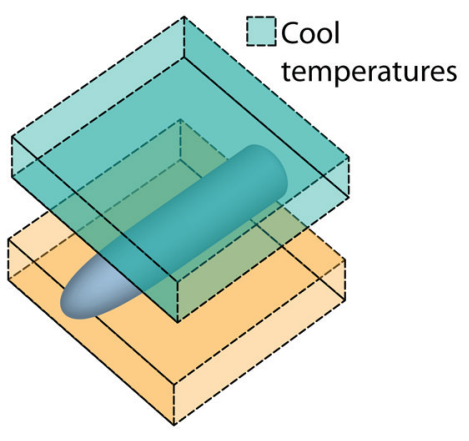

D)

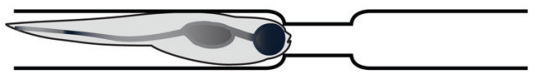

B)

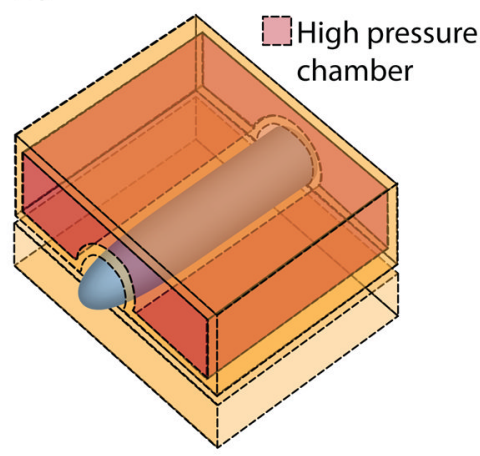

E)

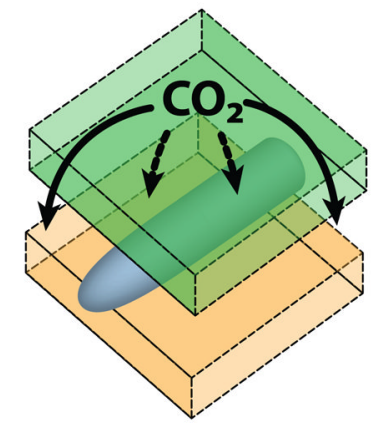

C)

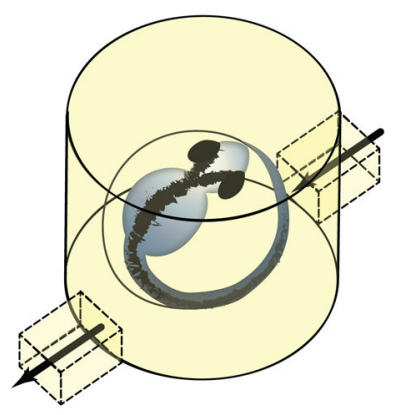

F)

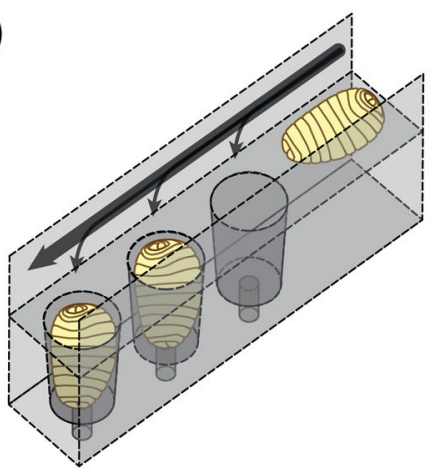

Figure 2.

General techniques for multicellular organism manipulation. A) Immobilization of animals using local cooling. B) Immobilization of animals using a high-pressure valve to wrap around the animal and trap it. C) Perfusion chambers for long-term growth and observation. D) Using confined spaces to constrict animals to prevent movement and align animals. E) Employing gas diffusion through a thin membrane in conjunction with physical constriction to immobilize animals. F) Using fluid flow to orient embryos in high-density arrays. 